\title{
Are newly graduated nurses ready to deal with death and dying? - A literature review
}

\author{
Linda Deravin Malone*, Judith Anderson and Lyn Croxon \\ School of Nursing, Midwifery and Indigenous Health, Charles Sturt University, Australia
}

\begin{abstract}
Objectives: This article examines the literature currently available to explore the readiness of students and new graduate nurses when faced with death and dying within the workplace.

Design: This study was a systematic literature review on research articles from peer reviewed journals using the PRISMA framework.

Data sources: Databases utilised in the search included EBSCOHost, Primo Search and Google Scholar.

Review methods: Key search terms included new graduate nurses, life limiting illness, nursing undergraduate, nursing students, palliative care. Abstracts of these articles were reviewed to ensure that they related to new graduate and undergraduate nurse experiences with death and dying. Further interrogation of the reference lists located another 39 articles for possible inclusion. Duplicates were removed. Articles for inclusion were to be full text articles available in English and within the date range of 2009 to 2015 . This left a total of 31 articles for the review.

Results: From the review four key themes emerged. These were; the importance of palliative care in undergraduate nursing curricula, readiness for dealing with death and dying, the death experience for different patient populations and education strategies.

Conclusions: There is an increasing emphasis on education strategies to assist with the end of life care knowledge and skills for nursing students. Simulation is seen to be a positive way to provide undergraduate education in end of life care as simulated exposure to dying patients is recognised as an effective means alleviating anxiety regarding death and the care of dying patients. While palliative care is viewed as an important aspect of undergraduate nursing education it is recognised as an area of practice that undergraduate nurses feel they are not adequately prepared for. Undergraduate education needs to incorporate skills such as having conversations and communicating effectively with patients and families experiencing end of life issues.
\end{abstract}

\section{Introduction}

Today, advances in medical care have led to the medicalization of end of life care with the majority of people dying in acute care settings rather than their preference to die in the familiar environment of home. Around half of deaths in Australia occur in acute care hospitals, the majority being over 75 years of age [1]. Around one third of New Zealand deaths occur in acute hospitals and one third in the home [2]. There are a range of health professionals that provide care to people who have a life limiting condition; however nurses spend more time with people nearing the end of their life, than any other health professional. For this reason, it is important that graduate nurses have core capabilities required to care for these people [3-6]. Nursing students agree that palliative care is an essential component of their curriculum $[7,8]$.

\section{Objectives}

This article examines the literature currently available to explore the readiness of students and new graduate nurses when faced with death and dying within the workplace.

\section{Design}

This study was a systematic literature review on research articles from peer reviewed journals following the PRISMA framework.

\section{Data sources}

Databases utilised in the literature search included EBSCOHost, Primo Search and Google Scholar. Once the initial yield of article was reviewed, reference lists were scrutinised to identify further articles for possible inclusion.

\section{Review methods}

For the systematic literature review, key search terms included: new graduate nurses, life limiting illness, nursing undergraduate, nursing students, palliative care. This initial search yielded a total of 32 research articles from peer reviewed journals. Abstracts of these articles were reviewed by the researchers to ensure that they met the inclusion criteria of being; research articles related to the topic of enquiry, were within the specified date range, and were related to new graduate and undergraduate nurse experiences with death and dying.

Correspondence to: Linda Deravin Malone, RN, MHM, BN, FACHSM, Lecturer in Nursing, Charles Sturt University, School of Nursing, Midwifery and Indigenous Health, Panorama Ave, Bathurst, NSW, 2795, Australia; E-mail: lmalone@csu.edu.au

Key words: new graduate nurses, palliative care, death and dying, undergraduate nurse curriculum

Received: July 02, 2016; Accepted: July 22, 2016; Published: July 27, 2016 
From the first yield of articles further interrogation of the reference lists located a further 39 articles for possible inclusion. Once duplicates were removed a total of 58 articles remained which were then screened. Articles were limited to the date range of 2009 to 2015, and full text articles available in English, which left a total of 31 articles. All articles to be included in the review were then read and summarised in Figure 1.

\section{Results}

From the review of the 31 articles included in this systematic literature review, four key themes emerged. These were; the importance of palliative care in undergraduate nursing curricula, readiness for dealing with death and dying, the death experience for different patient populations and education strategies. These themes are further described below.

\section{The importance of palliative care in undergraduate nursing curricula}

Several authors indicate that good quality education related to palliative care is included in undergraduate nursing curricula worldwide [8,9], with some also suggesting that student outcomes included a positive perspective to working with these people [8]. However, other studies indicate a recognition of the need to increase palliative care education in undergraduate nursing curricula $[8,10]$ and indicate that undergraduate nursing students are often unprepared to provide end-of-life care [8]. A survey of 41 Australian universities by Hegarty et al. [11], found although the content areas being taught were shared the quantity of time devoted to palliative care and the teaching and learning approaches varied across all universities leading them to conclude that exposure to end-of-life care content is limited in Australian undergraduate health service curricula. A study involving $82 \%$ of universities in Canada found all but one provided end of life education [9]. Although there was an overall increase in the amount of class time devoted to the topic from 2004 to 2010, another Canadian studies [12] describe nursing, medical and pharmacology courses as being inadequate with regard to palliative care content and support the need to integrate palliative care knowledge into undergraduate curricula.

There is debate around whether palliative care content should be integrated across the curriculum or be a distinct course in a program. Al Qadire [12] conducted a study on the perceptions, knowledge and understanding of undergraduate nursing students in relation to palliative care. Their recommendation is that palliative care education needs to be incorporated within nursing courses and should cover care and management across the entire life spectrum so that nurses are prepared for practice. Wallace et al. [14] indicated a substantial need to integrate content across the curriculum. Bush and Shahwan-Akl [10], reported on Australian students in an elective palliative care subject who expressed the view that palliative care should be compulsory within undergraduate nursing courses. Dobbins [15] compared the attitudes of students who undertook an elective palliative care course with those who attended a 3-hour lecture embedded in their course. It was found that both methods of delivery of content had a positive effect
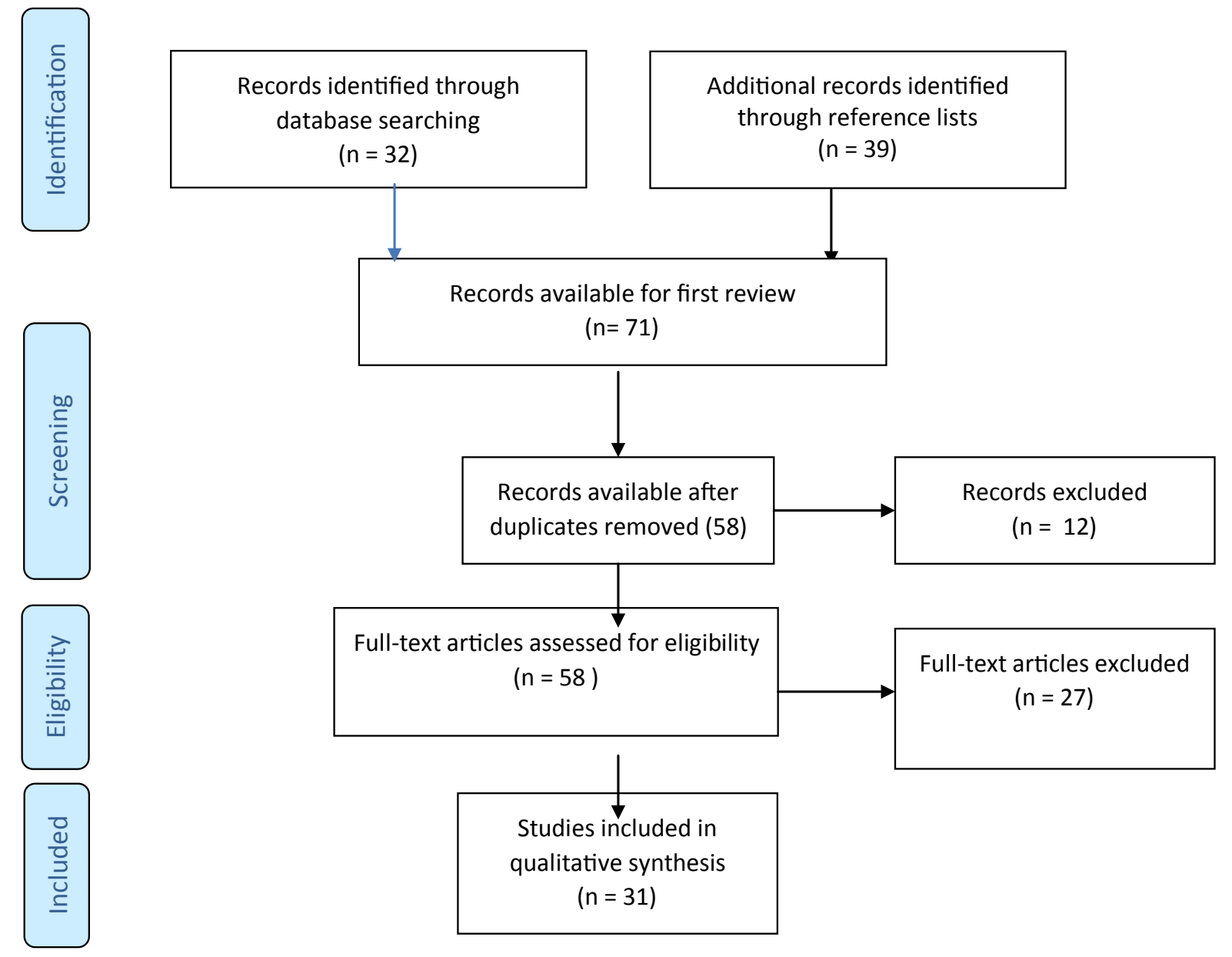

Figure 1. PRISMA Flow Diagram- Are new grads ready to deal with death and dying. 
on the attitude of students toward death and those who are dying and enhanced their ability to care for the physical, psychological, social, and spiritual needs of these people. There is also recognition of the need for skilled academics to teach into these areas and clinical placements that are specific to the practice of palliative care [10].

\section{Readiness for dealing with death and dying}

Despite palliative care being viewed as an important component of undergraduate nursing education it is an element that is also recognised as an area of practice that undergraduate nurses feel they are not adequately prepared for. This appears to be a global phenomenon revealed by studies in various countries including, India [16], Australia $[10,11,17]$, and the United States of America [15,18]. An area of concern that is raised amongst a number of studies [11,19] is that students do not realise they are unprepared to address this area of nursing until they are in practice. They do not know what they do not know. Bush and Shahwan-Akl [10] surveyed nursing students re their perceived ability in delivering end of life care after undertaking an elective course as part of their undergraduate educational program and found that they appreciated the importance of the course to their clinical practice and felt that there should be more emphasis on knowing how to nurse the palliative person.

Studies support the more exposure students have to death and end of life care the more positive their attitudes are to caring for these patients. Mutto et al. [20] conducted a study which examined the need for education in palliative care in Argentina and the importance of incorporating this education into undergraduate programs. In this study they recognised that nurses who had more exposure to death and dying patients through years of practice tended to have positive attitudes towards death and the care of dying patients. In this study only $3 \%$ of students had undergone any palliative care training. Occasional contact with dying patients did not change personal perception of suffering and the experience of death and recommended that more training is required to manage this part of the life cycle. Garity [21], in an American study found that education around the dying process decreased registered nurses' anxiety of death and dying. Ballesteros et al. [7] in a study in the United Kingdom indicated that students appreciated the palliative care course for its capacity to afford a broad perspective of nursing while developing communication skills and contributing to their individual growth.

In nursing the person who is facing death, students need to know the physical requirements in relation to pain management and the provision of comfort to ease suffering yet as highlighted in a study by Dobbins [15] the need for the student to understand the emotional, spiritual, cultural and psychosocial aspects of caring for the dying person is also equally as important. Students need to also be aware that often the nursing care is also extended to the family of the dying person [22]. Addressing this gap in knowledge so that undergraduate nursing students are prepared for future practice would seem vital yet there is little evidence in the literature that this occurs.

\section{The death experience for different patient populations}

Another aspect to consider when examining the readiness for undergraduate nursing students to care for the person in their palliative stage of life is that the death experience is unique to each individual. A variety of other factors come in to play such as culture and religion and psychosocial aspects of care. Iranmanesh et al. [23] in their comparison of Iranian and Swedish nursing students, found that attitudes towards palliative care differed substantially across cultures and suggested that education on the topic needed to be individualised and culturally sensitive in order to address these issues. Ellman et al. [18] claim that although palliative care is recognised as being important, only passing consideration has been given to the aspects of spiritual and cultural care in these patients. In this American study [18] an interprofessional palliative care educational online package focussing on spiritual and cultural care of patients was found to promote interprofessional collaboration and increased emphasis on a team approach to care.

Simultaneously there is also a need for focus on different age groups. Lindsay [24] highlighted that most end of life care focuses on the care of the dying adult patient yet little is provided in the way of paediatric death. This leads to emotional stress in both students and nurses when dealing with paediatric patients. A way to address this gap is to support the undergraduate nursing student in a simulated and controlled environment where educators are available to monitor emotional reactions and provide strategies to address these reactions should they occur $[19,25]$.

\section{Education strategies}

There have been a variety of education strategies used to engage students in learning about end of life care. Simulation is presented by several authors as a useful strategy in preparing students for end of life scenarios [17,18,22,24,26-30], particularly as opportunities to care for these people while learning may be limited. Simulation was found to be effective in increasing knowledge, self-confidence $[26,28,29]$, self-reported communication skills and student satisfaction [26]. Students found real time patient status changes in simulation to be valuable in addition to providing a safe learning environment for improving technical skills $[27,28]$. Some authors $[17,29]$ indicate that simulation is useful as it assists in the recreation of an emotionally charged experience, but in a safe environment [17] which can lead to a reduction in anxiety amongst nursing students, especially those who are younger with limited experiences of death and grief [29]. Yildiz \& Akansel [31] support this, recommending that first year students not undertake placement in the clinical environment if there was a high possibility of caring for dying people. Hamilton [25] particularly indicates the need for reflection or debriefing after simulation to ensure that it is effective in reducing anxiety. This was supported by Ellman et al. [18] in a study which used an online live interactive simulation' to allow interdisciplinary students to reflect, particularly on the spiritual and cultural facets of end of life care. Eaton et al. [22] in their phenomenological study of simulation in end of life care found three themes of: experiential learning, affirmative outcomes and family which they felt affirmed the value of using simulation to teach end of life care.

Brajtman, Fothergill-Bourbonnais, Fiset, and Alain [32] indicate that meaningful learning experiences should involve team work and clinical decision making activities. In contrast Liu et al. [33], describe a reflective experience where the data of 20 Taiwanese students' journals was presented as they imagined themselves with only 30 mins to live. The nature of the reflection is presented as a suitable way to facilitate selfawareness, peer support and how to manage their emotions. Dobbins [15] identified the use of movies and movie clips, 'cinemeducation' (p.161) as a means of promoting reflection, and influencing attitudes by linking the experiences to feelings and beliefs for nursing students, which decreased anxiety, avoidance and negative feelings about dying and increased acceptance of death as a life stage. Gillan et al. [17] also describe the use of 'cinemeducation' which involves the use of feature films to engage students in thinking about grief, loss and the intention 


\section{of palliative care.}

The need to consider ethical theory and decision-making in the education of nursing students about end of life care is also described [21]. Garity feels this is necessary to avoid moral distress and retain nurses in practice and is also supported by external agencies which frequently accredit nursing courses. The need to address cultural sensitivity [23] and ethnic background [19] was also raised as an issue in relation to education strategies.

In a survey of 51 students who completed an elective palliative care subject Bush and Shahwan-Akl [10] students felt that palliative care should be compulsory in all undergraduate nursing courses, however, Klemp et al., [34] indicates that undergraduate courses were inadequate in this area and that postgraduate education was required to address this gap. Some authors $[13,14]$ warn against limiting education on end of life care to a single subject, but rather suggest that it is more effective to integrate this aspect of nursing education across the undergraduate course. However, Brajtman et al., [32] caution that educators in this area of expertise need time, opportunities and relevant resources to support student learning.

\section{Conclusion}

Australian undergraduate courses vary in terms of the nature and extent of education provided in palliative care. The literature supports a need to include good quality education relating to end of life care in undergraduate nursing curricula. End-of-life care content is limited in Australian courses and teaching and learning strategies. Globally, this is an area of practice that undergraduate nurses feel they are not adequately prepared for. With the majority of deaths in Australia occurring in health care facilities it is essential that nurses, including new graduate nurses have the essential skills and knowledge required to care for these people.

There is an increasing emphasis on education strategies to assist with the end of life care knowledge and skills for nursing students. Simulation is seen to be a positive way to provide undergraduate education in end of life care as simulated exposure to dying patients is recognised as an effective means alleviating anxiety regarding death and the care of dying patients. While palliative care is viewed as an important aspect of undergraduate nursing education it is recognised as an area of practice that undergraduate nurses feel they are not adequately prepared for. The literature identifies that gaps exist for practicing nurses and highlight that undergraduate education needs to incorporate skills such as having conversations and communicating effectively with patients and families experiencing end of life issues.

\section{Acknowledgement}

The funding for this review article is provided by CSU research seed grant.

\section{References}

1. Australian Institute of Health and Welfare (2015) Australian Burden of Disease Study: Fatal burden of disease 2010. (Cat. no. BOD 1). Canberra: AIHW.

2. Ministry of Health (2014) New Zealand Burden of Diseases, Injuries and Risk Factors Study, 2006-2016.

3. Anderson J, Malone L (2015) Chronic care undergraduate nursing education in Australia. Nurse Educ Today 35: 1135-1138. [Crossref]

4. Deravin-Malone L, Croxon L, McLeay M, Anderson J (2016) End of Life Care. In L. Deravin-Malone \& J. Anderson (Eds.), Chronic Care Nursing: A Framework for Practice. Melbourne: Cambridge University Press.
5. Palliative Care Australia (2005) A guide to palliative care service development: A population based approach. Canberra: Palliative Care Australia.

6. Ramjan JM, Costa CM, Hickman LD, Kearns M, Phillips JL (2010) Integrating palliative care content into a new undergraduate nursing curriculum: The University of Notre Dame, Australia - Sydney experience. Collegian 17: 85-91.

7. Ballesteros M, Centeno C, Arantzamendi M (2014) A qualitative exploratory study of nursing students' assessment of the contribution of palliative care learning. Nurse Educ Today 34: e1-6. [Crossref]

8. Mutto EM, Cantoni MN, Rabhansl MM, Villar MJ (2012) A perspective of end-oflife care education in undergraduate medical and nursing students in Buenos Aires, Argentina. J Palliat Med 15: 93-98. [Crossref]

9. Wilson DM, Goodwin BL, Hewitt JA (2011) An Examination of Palliative or End-ofLife Care Education in Introductory Nursing Programs across Canada. Nurs Res Pract 2011: 907172. [Crossref]

10. Bush T, Shahwan-Akl L (2013) Palliative care education: Does it influence future practice. Journal of Palliative Care \& Medicine 2.

11. Hegarty M, Currow D, Parker D, Turnbull B, Devery K, et al. (2010) Palliative care in undergraduate curricula: Results of a national scoping study. Focus on Health Professional Education: A Multi-disciplinary Journal 12: 97 -109.

12. Cheung WY, Fishman PN, Verma S (2009) Oncology education in Canadian undergraduate and postgraduate training programs. J Cancer Educ 24: 284-290. [Crossref]

13. Al Qadire M (2014) Knowledge of palliative care: an online survey. Nurse Educ Today 34: 714-718. [Crossref]

14. Wallace M, Grossman S, Campbell S, Robert T, Lange J, et al. (2009) Integration of end-of-life care content in undergraduate nursing curricula: student knowledge and perceptions. J Prof Nurs 25: 50-56. [Crossref]

15. Dobbins EH (2011) The impact of end-of-life curriculum content on the attitudes of associate degree nursing students toward death and care of the dying. Teaching and Learning in Nursing 6: 159-166.

16. Sadhu S, Salins NS, Kamath A (2010) Palliative care awareness among Indian undergraduate health care students: a needs-assessment study to determine incorporation of palliative care education in undergraduate medical, nursing and allied health. Indian Journal of Palliative Care 16: 154-159.

17. Gillan PC, Parmenter G, van der Riet PJ, Jeong S (2013) The experience of end of life care simulation at a rural Australian University. Nurse Educ Today 33: 1435-1439. [Crossref]

18. Ellman MS, Schulman-Green D, Blatt L, Asher S, Viveiros D, et al. (2012) Using online learning and interactive simulation to teach spiritual and cultural aspects of palliative care to interprofessional students. Journal of Palliative Medicine 15: 1240-1247.

19. Range LM, Rotherham AL (2010) Moral distress among nursing and non-nursing students. Nurs Ethics 17: 225-232. [Crossref]

20. Mutto EM, Errazquin A, Rabhansl MM, Villar MJ (2010) Nursing education: The experience, attitudes, and impact of caring for dying patients by undergraduate Argentinian nursing students. Journal of Palliative Medicine 13: 1445-1450.

21. Garity J (2009) Fostering nursing students' use of ethical theory and decision-making models: teaching strategies. Learning in Health \& Social Care 8: 114-122.

22. Eaton MK, Floyd K, Brooks S (2012) Student perceptions of simulation's influence on home health and hospice practicum learning. Clinical Simulation In Nursing 8 : e239-e247.

23. Iranmanesh, Axelsson K, Häggström T, Sävenstedt S (2010) Caring for dying people: attitudes among Iranian and Swedish nursing students. Indian J Palliat Care 16: 147153. [Crossref]

24. Lindsay $\mathbf{J}$ (2010) Introducing nursing students to pediatric end-of-life issues using simulation. Dimens Crit Care Nurs 29: 175-178. [Crossref]

25. Hamilton C (2010) The simulation imperative of end-of-life education. Clinical Simulation in Nursing 6: e131-e138.

26. Fluharty E, Hayes AS, Milgrom LKM, Smith D, Reklau MA, et al. (2012) A multisite, multieacademic track evaluation of end-of-life simulation for nursing education. Clinical Simulation in Nursing 8: e135-e145.

27. Garrett B, MacPhee M, Jackson C (2010) High-fidelity patient simulation considerations for effective learning. Nurs Educ Perspect 31: 309-313. [Crossref] 
28. Leighton K, Dubas J (2009) Simulated death: An innovative approach to teaching endof-life care. Clinical Simulation in Nursing 5: e223-e230.

29. Moreland SS, Lemieux ML, Myers A (2012) End-of-life care and the use of simulation in a baccalaureate nursing program. Int J Nurs Educ Scholarsh 9. [Crossref]

30. Smith-Stoner M (2009) Using high-fidelity simulation to educate nursing students about end-of-life care. Nurs Educ Perspect 30: 115-120. [Crossref]

31. Yildiz H, Akansel N (2011) Beginning level nursing students' experiences with cancer patients in their first clinical placement: a qualitative appraisal in Turkey. Asian Pacific Journal of Cancer Prevention 12: 2611-2615.
32. Brajtman S, Fothergill-Bourbonnais F, Fiset V, Alain D (2009) Survey of educators' end-of-life care learning needs in a Canadian baccalaureate nursing programme. International Journal of Palliative Nursing 15: 233-241.

33. Liu YC, Su PY, Chen CH, Chiang HH, Wang KY, et al. (2011) Facing death, facing self: nursing students' emotional reactions during an experiential workshop on life-anddeath issues. Journal of Clinical Nursing 20: 856-863.

34. Klemp JR, Frazier LM, Glennon C, Trunecek J, Irwin M (2011) Improving cancer survivorship care: oncology nurses' educational needs and preferred methods of learning. Journal of cancer education: the official journal of the American Association for Cancer Education 26: 234-242.

Copyright: $\odot 2016$ Malone LD. This is an open-access article distributed under the terms of the Creative Commons Attribution License, which permits unrestricted use, distribution, and reproduction in any medium, provided the original author and source are credited. 